\title{
The accuracy of prostate cancer diagnosis in biopsy-naive patients using combined magnetic resonance imaging and transrectal ultrasound fusion-targeted prostate biopsy
}

\author{
Hiromi Uno ${ }^{1}$, Tomoki Taniguchi ${ }^{1}$, Kensaku Seike ${ }^{1}$, Daiki Kato ${ }^{2}$, Manabu Takai $^{2}, K_{\text {Koji Iinuma }}{ }^{2}$, \\ Kengo Horie ${ }^{2}$, Keita Nakane ${ }^{2}$, Takuya Koie ${ }^{2}$ \\ ${ }^{1}$ Department of Urology, Chuno Kosei Hospital, Seki, Japan; ${ }^{2}$ Department of Urology, Gifu University Graduate School of Medicine, Gifu, Japan \\ Contributions: (I) Conceptualization: H Uno; (II) Administrative support: T Koie; (III) Provision of study materials or patients: All authors; (IV) \\ Collection and assembly of data: All authors; (V) Data analysis and interpretation: All authors; (VI) Manuscript writing: All authors; (VII) Final \\ approval of manuscript: All authors. \\ Correspondence to: Takuya Koie. Department of Urology, Gifu University Graduate School of Medicine, 1-1 Yanagido, Gifu, Japan. \\ Email: goodwin@gifu-u.ac.jp.
}

\begin{abstract}
Background: This study aimed to estimate whether multiparametric magnetic resonance imaging (mpMRI)-transrectal ultrasound (TRUS) fusion biopsy (FUS-TB) increases the detection rates of clinically significant prostate cancer (csPCa) compared with TRUS-guided systematic biopsy (TRUS-GB).
\end{abstract}

Methods: This retrospective study focused on patients who underwent mpMRI before prostate biopsy (PB) with Prostate Imaging Reporting and Data System version 2 (PI-RADS v2) scores $\geq 3$ and prostate-specific antigen (PSA) level between 2.5 and $20 \mathrm{ng} / \mathrm{mL}$. Before FUS-TB, the biopsy needle position was checked virtually using three-dimensional mapping. After confirming the position of the target within the prostate, biopsy needle was inserted and PB was performed. Suspicious lesions were generally targeted with 2 to 4 cores. Subsequently, 10-12 cores were biopsied for TRUS-GB. The primary endpoint was the PCa detection rate (PCDR) for patients with PCa who underwent combined FUS-TB and TRUS-GB.

Results: According to PI-RADS v2, 76.7\% of the patients with PI-RADS v2 score $\geq 3$ were diagnosed with PCa. The PCDRs in patients with PI-RADS v2 score of 4 or 5 were significantly higher than those in patients with PI-RADS v2 score of 3 (3 vs. 4, $\mathrm{P}<0.001 ; 3$ vs. $5, \mathrm{P}<0.001 ; 4$ vs. 5, $\mathrm{P}=0.073$ ). According to PCDR, the detection rates of $\mathrm{PCa}$ and $\mathrm{cs} \mathrm{PCa}$ in the FUS-TB were significantly higher than that in the TRUS-GB.

Conclusions: Following detection of suspicious tumor lesions on mpMRI, FUS-TB use detects a higher number of PCa cases compared with TRUS-GB.

Keywords: Prostate cancer detection (PCa detection); fusion biopsy; magnetic resonance imaging (MRI); targeted biopsy

Submitted Mar 22, 2021. Accepted for publication Jun 09, 2021.

doi: $10.21037 /$ tau-21-250

View this article at: https://dx.doi.org/10.21037/tau-21-250

\section{Introduction}

Prostate cancer $(\mathrm{PCa})$ is the fourth most common cancer diagnosed and the sixth most common cause of cancer death in Japanese males (1). To date, screening for PCa using prostate-specific antigen (PSA), digital rectal examination (DRE), and transrectal ultrasound (TRUS), based on several guidelines, remains controversial (2). Although the PSA test is widely used and seems to increase the detection of PCa of any stage (3), it has a poor specificity for clinically significant PCa (csPCa); hence, approximately $60-70 \%$ of patients with increased PSA levels who underwent initial biopsies have negative PCa results (4). Additionally, up to $45 \%$ of 
patients diagnosed with $\mathrm{PCa}$ are at low-risk according to the D'Amico risk stratification $(2,5)$. The use of DRE as a screening modality is not recommended because there is a lack of evidence regarding its benefits. Therefore, DRE was not included in the major screening trials (6). Furthermore, the presence of hypoechoic lesions on TRUS is not always specific to PCa; therefore, the accuracy of TRUS in diagnosing PCa relies on the operator's knowledge and experience (7-9).

TRUS-guided systemic biopsy (TRUS-GB) through the transperineal route is one of the standard techniques for detecting $\mathrm{PCa}$ and avoiding $\mathrm{PB}$-related infectious complications. The technique can help collect 10-14 template cores of the prostate (10-12). However, TRUS-GB is known for its low sensitivity and specificity in detecting $\mathrm{PCa}$, with an overall cancer detection rate of $27-40.3 \%(2,13)$. Furthermore, 750,000 men diagnosed with suspicious PCa using the aforementioned screening modalities underwent unnecessary biopsies, with the accompanying pain, inconvenience, financial burden, and risk of infection (14). Therefore, it is necessary to identify and improve alternate methods of diagnosis in patients with positive predictive values so as to avoid unnecessary biopsies (15).

Multiparametric magnetic resonance imaging (mpMRI), including T2-weighted imaging (T2WI), diffusion-weighted imaging, and dynamic contrast material enhancement, is a powerful tool for identifying csPCa $(12,16,17)$. Additionally, in 2012, the European Society of Urogenital Radiology recommended using the Prostate Imaging Reporting and Data System (PI-RADS) to determine whether lesions detected by mpMRI reflect PCa risk (18). In 2015, PI-RADS version 2 (PI-RADS v2) simplified the rules for reporting modified imaging sequences for identifying csPCa (19). Men who had positive results on the mpMRI underwent MRItargeted biopsy with the use of real-time ultrasonographic guidance (20). However, it is possible to miss csPCa using MRI-TRUS fusion targeted biopsy (FUS-TB) alone (21).

Thus, this study aimed to estimate whether FUS-TB increases the detection rates of csPCa compared with TUR-GB.

We present the following article in accordance with the STARD reporting checklist (available at https://dx.doi. org/10.21037/tau-21-250).

\section{Methods}

\section{Patients}

This retrospective study enrolled 580 consecutive patients who had undergone mpMRI before prostate biopsy (PB) from February 2015 to February 2020 at Chuno Kosei Hospital. We reviewed their clinical and pathological records, focusing on PI-RADS v2 scores $\geq 3$ and serum PSA levels in the range $2.5-20 \mathrm{ng} / \mathrm{mL}$ (19). According to the 2010 American Joint Committee on Cancer Staging Manual, we excluded patients with lymph node involvement, distant metastases, or clinical stage T4 tumors from the study (22). We also excluded patients with urinary tract infections, those who had undergone PB before 2014, and those with a serum PSA level of $>20 \mathrm{ng} / \mathrm{mL}$.

The study was conducted in accordance with the Declaration of Helsinki (as revised in 2013). The study protocol was approved by the Institutional Review Board of Chuno Kosei Hospital (R2-7) and Gifu University (30-031) and informed consent was taken from all the patients.

\section{mpMRI protocol}

Prior to $\mathrm{PB}, \mathrm{mpMRI}$ was performed with Phillips Achieva 1.5 Tesla system (Philips Medical Systems, Best, The Netherlands) using a 5-channel sensitivity Encoding parallel imaging cardiac coil covering the entire prostate. PI-RADS v2 criteria were used to describe the mpMRI findings, and this was done by two radiologists with more than 10 years of clinical experience each.

\section{Prostate biopsy protocol}

A cleansing enema and antibiotics were used before biopsy in all patients. An $18-\mathrm{G} \mathrm{BARD}^{\circledR} \mathrm{MAX}^{-\mathrm{CORE}^{\circledast}}$ disposable core biopsy instrument (C.R. Bard Medical, United States) was used to perform TRUS-GB under local anesthesia. Transrectal PB, including TRUS-GB and FUS-TB, was performed by an experienced single urologist (HU) using Urostation ${ }^{\circledR}$ between February 2015 and March 2018 or using TRINITY ${ }^{\circledR}$ between April 2018 and February 2020 (Koelis, Meylan, France) with the three-dimensional (3D)TRUS-based biopsy mapping technique. MRI images were imported into the Urostation ${ }^{\circledR}$ or TRINITY ${ }^{\circledR}$. The contour of the prostate was delineated on T2WI, and a target was marked using the resizable circle. Following DRE, the 3D-TRUS probe (end-fire 3D-transrectal transducer) was inserted into the rectum. The entire prostate was scanned by 3D-TRUS, with the probe kept in the same place, and the image created was saved in the workstation. Subsequently, the probe was moved from the apex of the prostate to the base and from side to side until an angle of $60^{\circ}$ was covered 

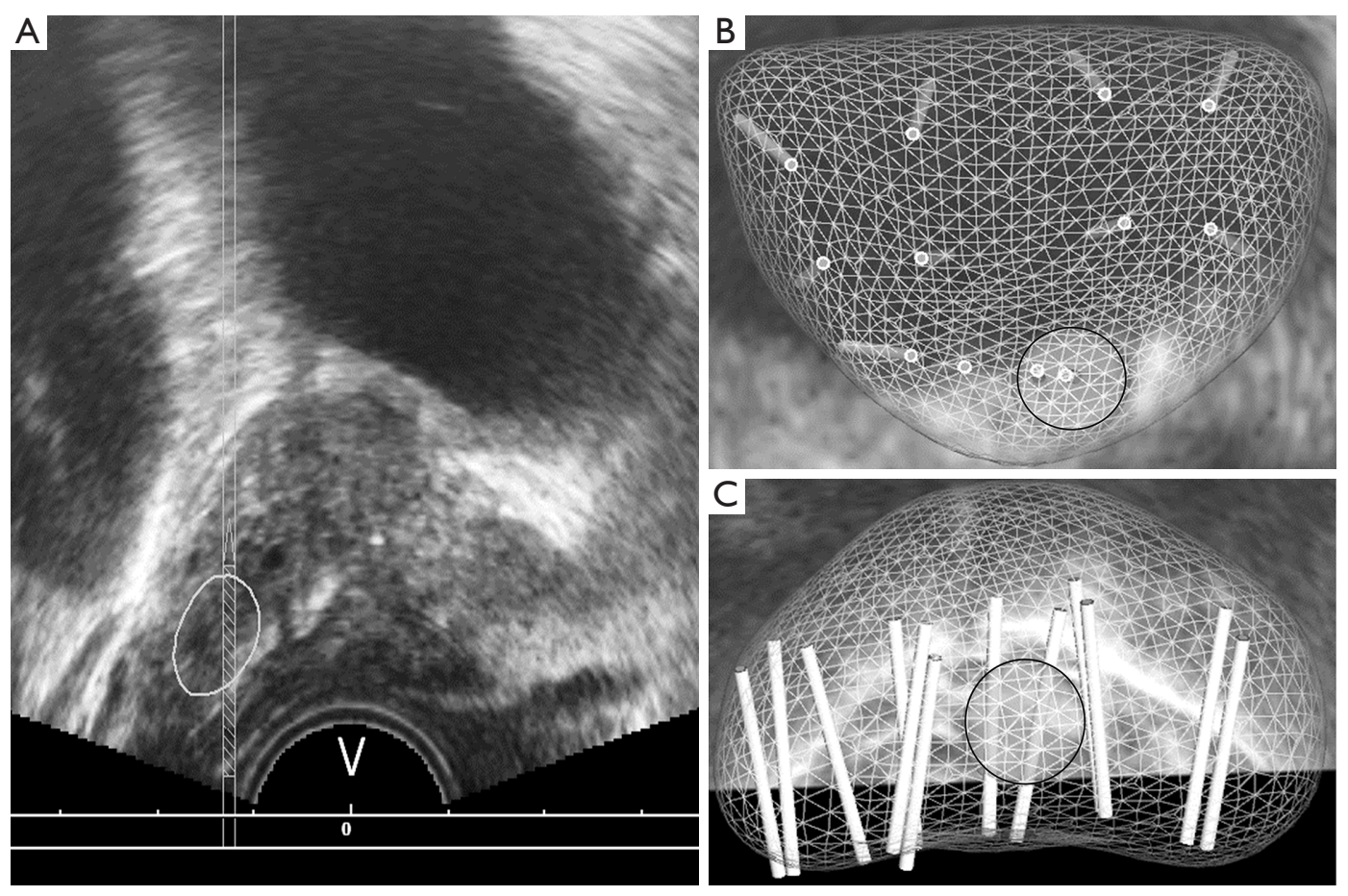

Figure 1 The method of multiparametric magnetic resonance imaging (mpMRI)-transrectal ultrasound fusion targeted biopsy (FUS-TB). (A) Before FUS-TB, biopsy needle position was checked virtually using three-dimensional (3D) mapping. (B,C) The position of the collected biopsy core was checked again using the 3D mapping (B, axial view; $\mathrm{C}$, sagittal view).

in all direction. This provided a panoramic view of the entire prostate. Before FUS-TB, the biopsy needle position was checked virtually using 3D mapping (Figure 1A). After confirming the position of the target within the prostate, the biopsy needle was inserted and PB was performed. The position of the biopsy core from which the sample is to be collected was checked again using $3 \mathrm{D}$ mapping (Figure 1B,C). If the biopsy core did not penetrate the center of the target, we performed a re-biopsy at a similar position. For suspicious lesions on biparametric MRI (PI-RADS v2 scores $\geq 3$ ), 2-4 targeted cores were usually selected depending on lesion size. Subsequently, we obtained 10-12 cores-including medial and lateral cores from bilateral sextant prostate regions where no indications of $\mathrm{PCa}$ were noted on mpMRI-for TRUS-GB. The complications according to the type of $\mathrm{PB}$ were evaluated according to the Clavien-Dindo classification (23).

\section{Pathological analysis}

The PB specimens were evaluated by a single pathologist at Chuno Kosei Hospital according to the 2005 International
Society of Urological Pathology guidelines (24). The definition of csPCa was having at least one core with a Gleason score (GS) of $3+4$ or 6 and a maximum cancer core length (MCCL) $\geq 5 \mathrm{~mm}$. Insignificant cancer was defined as having a core with a GS $\leq 6$ and an MCCL $\leq 4 \mathrm{~mm}$ (25).

\section{Endpoints}

Based on the PI-RADS v2 criteria, the PCa detection rate (PCDR) on mpMRI was defined as the primary endpoint in patients who were suspected of having $\mathrm{PCa}$ (i.e., csPCa or clinically insignificant $\mathrm{PCa}$ ) via the combined FUS-TB and TRUS-GB. Based on the PI-RADS v2 score and GS obtained from the $\mathrm{PB}$ samples, the detection rate of csPCa was defined as the secondary endpoint.

\section{Statistical analysis}

Data were analyzed using the Statistical Package for the Social Sciences software version 19.0 (International Business Machines Corp., Armonk, NY, USA). We used Student's $t$-test or Mann-Whitney $\mathrm{U}$ test and the McNemar test to 


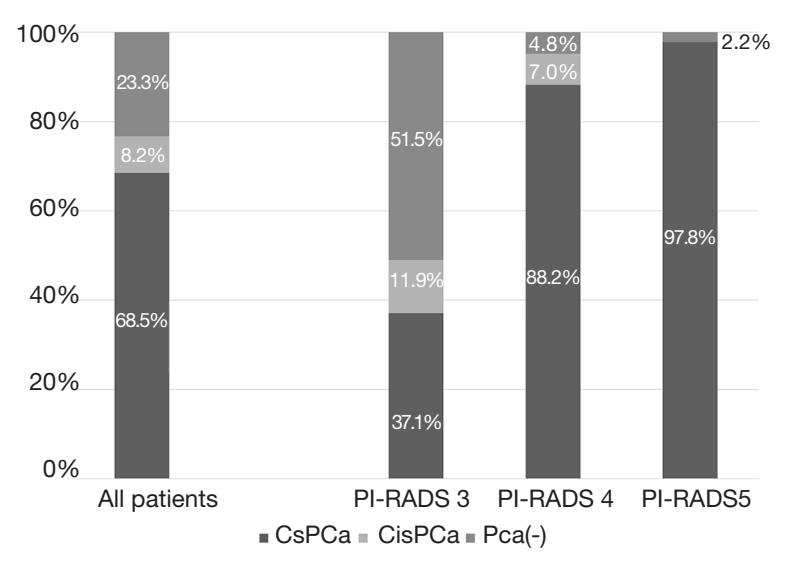

Figure 2 According to the Prostate Imaging Reporting and Data System version 2 (PI-RADS v2), 76.7\% of patients with PI-RADS v2 score $\geq 3$ were diagnosed with $\mathrm{PCa}$. The PCDRs in patients with PI-RADS v2 score of 4 or 5 were significantly higher than those with PI-RADS v2 score of 3 ( 3 vs. $4, \mathrm{P}<0.001 ; 3$ vs. 5, $\mathrm{P}<0.001$; 4 vs. 5, $\mathrm{P}=0.073)$.

compare continuous variables and categorical variables, respectively. All $\mathrm{P}$ values were two-sided, and statistical significance was set at $\mathrm{P}<0.05$.

\section{Results}

\section{Patient demographic}

This study enrolled a total of 391 patients, 300 of whom $(76.7 \%)$ were diagnosed as having PCa after PB. The median age and initial PSA level were 71 years [interquartile range (IQR), $67-77$ years] and $6.03 \mathrm{ng} / \mathrm{mL}$ (IQR, 4.62-8.96 ng/mL), respectively. Abnormal DRE was observed in $66(16.9 \%)$ patients. According to PI-RADS v2 criteria, 159 (40.7\%), 186 (47.6\%), and 46 (11.7\%) patients were diagnosed with category 3,4 , and $5 \mathrm{PCa}$, respectively.

All the complications related to $\mathrm{PB}$ were $\leq$ grade 2 according to the Clavien-Dindo classification. None of the patients died or was readmitted due to $\mathrm{PB}$.

\section{Comparison of cancer detection}

PCDRs according to the PI-RADS v2 criteria are shown in Figure 2. A total of $76.7 \%$ of the patients with PI-RADS v2 score $\geq 3$ was diagnosed with PCa. The PCDRs in patients with PI-RADS v2 score of 4 or 5 were significantly higher than those in patients with PI-RADS v2 of 3 ( 3 vs. $4, \mathrm{P}<0.001 ; 3$ vs. $5, \mathrm{P}<0.001 ; 4$ vs. $5, \mathrm{P}=0.073)$. Of these,
113 patients (37.7\%) were diagnosed with PCa using FUSTB alone, while 43 patients (14.3\%) who tested negative in FUS-TB were left undiagnosed. The PCDR of csPCa was $46 \%$ for those who underwent combined FUS-TB and TRUS-GB.

Table 1 shows the comparison between FUS-TB and TRUS-GB for PCDR. The detection rates of PCa and csPCa following FUS-TB were significantly higher than that those following TRUS-GB.

\section{The association between Gleason score and the method of prostate biopsy regarding clinically significant $\mathrm{PCa}$}

Details of the patients diagnosed with csPCa using $\mathrm{PB}$ samples are shown in Table 2. Following PB, the concordance rate of both csPCa and GS or no evidence of PCa was $48.8 \%$. FUS-TB was associated with a significantly lower rate of diagnosis than the TRUS-GB (30.0\% vs. $13.6 \%$, respectively, $\mathrm{P}<0.001)$. Additionally, 55 patients (18.3\%) diagnosed with negative PCa underwent TRUSGB alone, although csPCa could not be overlooked in 12 patients (4.7\%) undergoing FUS-TB alone.

\section{Discussion}

It is still controversial whether the use of either FUS-TB or TRUS-GB alone has the ability to diagnose PCa in biopsynaïve patients with suspicious lesions on MRI. Hansen et al. showed that the combined use of FUS-TB and TRUS-GB is significantly superior to either FUS-TB or TRUS-GB alone in detecting $\mathrm{PCa}$ in patients with a GS of 7-10 and a PI-RADS v2 score of 4 or $5(\mathrm{P}<0.001)$ (26). For three lesions based on the PI-RADS score, the detection rate of csPCa was lower with FUS-TB than that with combined FUS-TB and TRUS-GB ( $\mathrm{P}=0.063)$, with no significant difference (26). Mannaerts et al. showed that the combined FUS-TB and TRUS-GB improved PCDRs from $5 \%$ to $15 \%$ when compared with TRUS-GB alone (27). A definitive diagnosis of unilateral disease was made via mpMRI in $22 \%$ of patients positive for csPCa with combined FUSTB and TRUS-GB, whereas bilateral csPCa was detected with FUS-TB alone (27). Although TRUS-GB is highly sensitive for characterizing index lesions, it often misses the detection of secondary lesions (27). TRUS-GB did not contribute to considerable changes in the PCDR $(\mathrm{P}>0.9)$ or the detection rate of $\operatorname{cs} \mathrm{PCa}(\mathrm{P}=0.67)$, although the PCDR and the csPCa detection rate with TRUS-GB were $51 \%$ (27 patients) and $82 \%$ (22 patients), respectively. Additionally, 
Table 1 Comparison of cancer detection between two groups

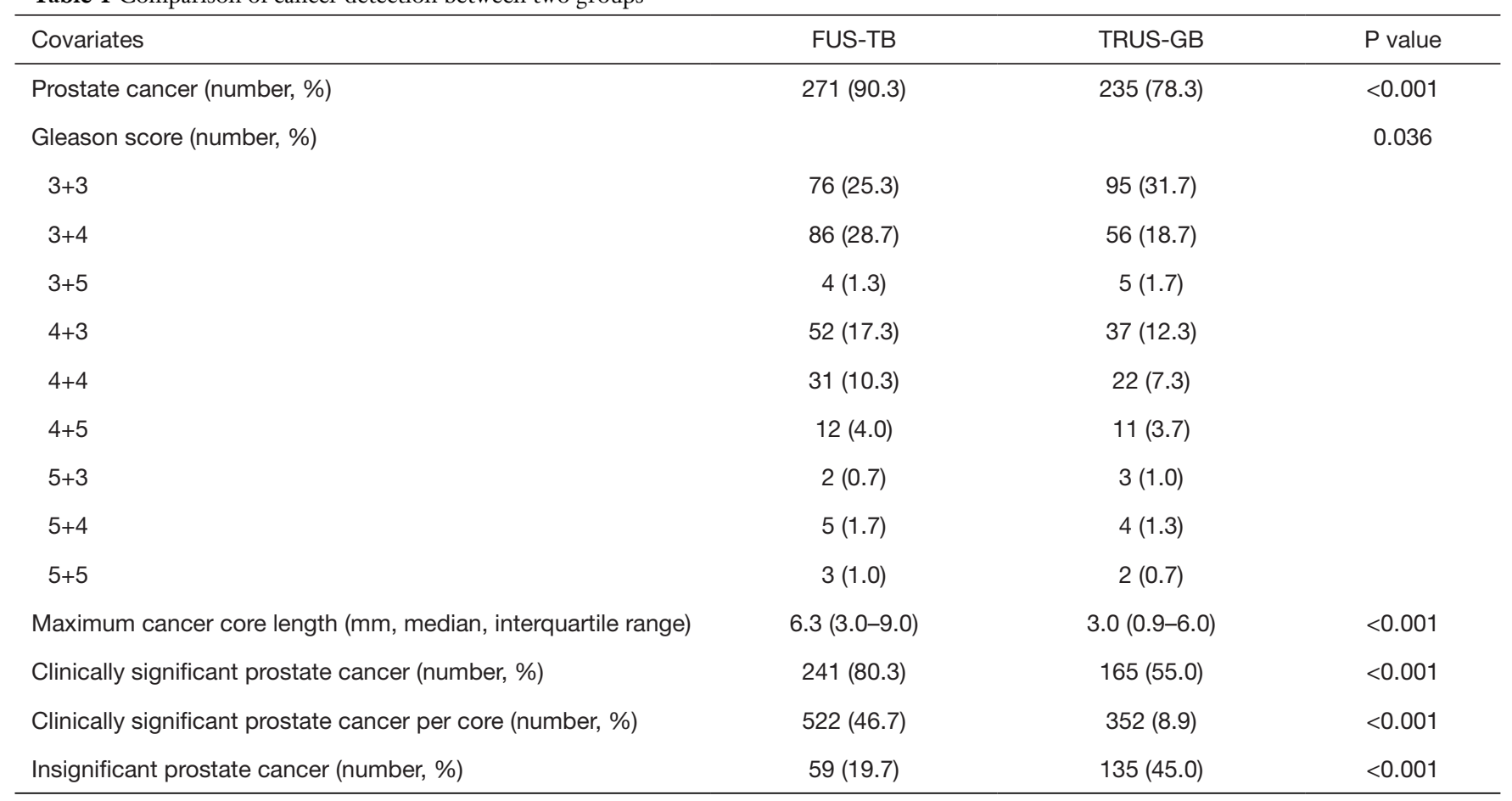

FUS-TB, magnetic resonance imaging-transrectal ultrasound fusion targeted biopsy; TRUS-GB, transrectal ultrasound guided systematic biopsy.

Table 2 The association between Gleason score and the method of prostate biopsy regarding clinically significant prostate cancer

\begin{tabular}{|c|c|c|c|c|c|}
\hline GS & \multicolumn{5}{|c|}{ FUS-TB (number, \%) } \\
\hline Negative & $91(23.3)$ & $11(2.8)$ & $32(8.2)$ & $9(2.3)$ & $3(0.8)$ \\
\hline 6 & $1(0.3)$ & $32(8.2)$ & $35(9.0)$ & $4(1.0)$ & $1(0.3)$ \\
\hline 7 & $11(2.8)$ & $13(3.3)$ & $51(13.0)$ & $12(3.1)$ & $7(1.8)$ \\
\hline 9,10 & $1(0.3)$ & $1(0.3)$ & $6(1.5)$ & $2(0.5)$ & $7(1.8)$ \\
\hline
\end{tabular}

FUS-TB, magnetic resonance imaging-transrectal ultrasound fusion targeted biopsy; TRUS-GB, transrectal ultrasound guided systematic biopsy; GS, Gleason score.

the detection rates of csPCa and clinically insignificant PCa with FUS-TB were $36 \%$ and $15 \%$, respectively (28). The combined use of FUS-TB and TRUS-GB produced a $20 \%$ reduction in risk of increased GS based on final histopathology (29). Interestingly, among patients initially diagnosed with low-risk PCa via TRUS-GB, $44.3 \%$ were subsequently diagnosed with intermediate-risk $\mathrm{PCa}$ via FUS-TB (29). In this study, using mpMRI images as the reference standard, combined FUS-TB and TRUS-GB had a significantly higher diagnostic accuracy for csPCa than TRUS-GB alone. Moreover, higher PCDR was noted for targeted cores than for systemic cores. To date, combined FUS-TB and TRUS-GB is considered necessary for the detection of csPCa.

It is important to evaluate the necessity of $\mathrm{PB}$ and use noninvasive imaging to guide biopsy location (30). The prebiopsy mpMRI may have several potential advantages in localizing PCa and noninvasively assessing risk; thus, this 
imaging modality is now routinely used in several clinical centers (12). Since real-time TRUS also visualizes an MRsuspicious lesion, the accuracy of biopsy targeting improved and the detection rate of csPCa thereby increased (7). It is known that the accurate interpretation of TRUS images relies on the operator's ability. However, the combined use of TRUS and mpMRI would increase their ability to visualize PCa, thereby enhancing the diagnostic accuracy of PCa. Thus, these two imaging modalities can compensate for lack of the operator's expertise or operator-dependency (7). Conversely, csPCa lesions that were not observed on MRI may have been underestimated than those that diagnosed csPCa on MRI (13). Consequently, MRI accuracy for PCa identification may differ between different centers and even between radiologists with different experiences and interpretation techniques (13). The Prostate MRI study trial reported a sensitivity of $93 \%$ and negative predictive value of $89 \%$ when identifying csPCa using mpMRI (31). However, Johnson et al. reported that mpMRI to detect csPCa using prostatectomy specimens as a reference standard had a lower sensitivity of $63 \%$ (32). A previous systemic review assessed the diagnostic accuracy of mpMRI for the detection of csPCa and found accuracy, sensitivity, and specificity ranges of $44-87 \%, 58-96 \%$, and $23-87 \%$, respectively (33). It is therefore important to make every effort to improve the precision of interpreting $\mathrm{mpMRI}$ and TRUS results towards developing a clinically significant FUS-TB (7). Additionally, the radiologist and urologist are required to share information regarding the imaging and biopsy results to arrive at a final diagnosis (7).

PI-RADS plays an essential role in detecting csPCa using mpMRI in order to select appropriate candidates for PB (17). Pooled meta-analyses with random-effect models showed that the positive predictive value for csPCa was $8 \%$ with PI-RADS 2, 13\% with PI-RADS 3, 40\% with PI-RADS 4, and $69 \%$ with PI-RADS 5 (34). Han et al. reported that the PCDR is $0 \%$ in patients with PI-RADS 1 and $4.6 \%$ in those with PI-RADS 2 (35). In our series, patients with PI-RADS 1 or 2 underwent TRUS-GB due to high PSA. The PCDR in these patients was $14.3 \%$ (data not shown). Additionally, approximately $90 \%$ of the patients did not undergo $\mathrm{PB}$ because they were diagnosed with PI-RADS 1 or 2 based on mpMRI findings. Indeed, PCa was undiagnosed in 30-48\% of patients as it was not visible on MRI (36). However, on the contrary, unnecessary $\mathrm{PB}$ was avoided in several patients with PI-RADS 1 or 2 in our series.

Our study has several limitations. First, this was a retrospective study with an inherent bias. Second, this was a nonrandomized study with no control group of patients. Third, as PB was not performed in all patients with PIRADS v2 scores of 1 or 2 , underestimation is possible. Fourth, a relatively high detection rate of csPCa was obtained in this study. Finally, in this study, we did not use prostatectomy specimens, and only focused on the correlation between mpMRI and biopsy pathology.

\section{Conclusions}

mpMRI is an emerging modality in the diagnosis, staging, and treatment planning of PCa. If suspicious tumor lesions are detected on mpMRI, the rate of diagnosis of PCa is higher with FUS-TB compared with TRUS-GB. Additionally, FUS-TB may improve risk stratification and reduce the need for repeat biopsy. Further comparative studies to identify the optimal methods for FUS-TB procedure, to evaluate cost-effectiveness, and to identify ways to avoid unnecessary $\mathrm{PB}$ are required.

\section{Acknowledgments}

Funding: None.

\section{Footnote}

Reporting Checklist: The authors have completed the STARD reporting checklist. Available at https://dx.doi. org/10.21037/tau-21-250

Data Sharing Statement: Available at https://dx.doi. org/10.21037/tau-21-250

Peer Review File: Available at https://dx.doi.org/10.21037/ tau-21-250

Conflicts of Interest: All authors have completed the ICMJE uniform disclosure form (available at https://dx.doi. org/10.21037/tau-21-250). The authors have no conflicts of interest to declare.

Ethical Statement: The authors are accountable for all aspects of the work in ensuring that questions related to the accuracy or integrity of any part of the work are appropriately investigated and resolved. The study was conducted in accordance with the Declaration of Helsinki (as revised in 2013). The study was approved by the Institutional Review Board of Chuno Kosei Hospital (R- 
7) and Gifu University (30-031) and informed consent was taken from all the patients.

Open Access Statement: This is an Open Access article distributed in accordance with the Creative Commons Attribution-NonCommercial-NoDerivs 4.0 International License (CC BY-NC-ND 4.0), which permits the noncommercial replication and distribution of the article with the strict proviso that no changes or edits are made and the original work is properly cited (including links to both the formal publication through the relevant DOI and the license). See: https://creativecommons.org/licenses/by-nc-nd/4.0/.

\section{References}

1. Project cancer statistics (2018) Available online: https:// ganjoho.jp/en/public/statistics/short_pred.html

2. Thompson J, Lawrentschuk N, Frydenberg M, et al. The role of magnetic resonance imaging in the diagnosis and management of prostate cancer. BJU Int 2013;112 Suppl 2:620.

3. Ilic D, Djulbegovic M, Jung JH, et al. Prostate cancer screening with prostate-specific antigen (PSA) test: a systematic review and meta-analysis. BMJ 2018;362:k3519.

4. Schröder FH, Carter HB, Wolters T, et al. Early detection of prostate cancer in 2007. Part 1: PSA and PSA kinetics. Eur Urol 2008;53:468-77.

5. D'Amico AV, Whittington R, Malkowicz SB, et al. Biochemical outcome after radical prostatectomy, external beam radiation therapy, or interstitial radiation therapy for clinically localized prostate cancer. JAMA 1998;280:969-74.

6. US Preventive Services Task Force; Grossman DC, Curry SJ, et al. Screening for Prostate Cancer: US Preventive Services Task Force Recommendation Statement. JAMA 2018;319:1901-13.

7. Ukimura O, Marien A, Palmer S, et al. Trans-rectal ultrasound visibility of prostate lesions identified by magnetic resonance imaging increases accuracy of imagefusion targeted biopsies. World J Urol 2015;33:1669-76.

8. Ukimura O, de Castro Abreu AL, Gill IS, et al. Image visibility of cancer to enhance targeting precision and spatial mapping biopsy for focal therapy of prostate cancer. BJU Int 2013;111:E354-64.

9. Ukimura O, Coleman JA, de la Taille A, et al. Contemporary role of systematic prostate biopsies: indications, techniques, and implications for patient care. Eur Urol 2013;63:214-30.

10. Wegelin O, van Melick HHE, Hooft L, et al. Comparing
Three Different Techniques for Magnetic Resonance Imaging-targeted Prostate Biopsies: A Systematic Review of In-bore versus Magnetic Resonance Imaging-transrectal Ultrasound fusion versus Cognitive Registration. Is There a Preferred Technique? Eur Urol 2017;71:517-31.

11. Roberts MJ, Bennett HY, Harris PN, et al. Prostate Biopsy-related Infection: A Systematic Review of Risk Factors, Prevention Strategies, and Management Approaches. Urology 2017;104:11-21.

12. Pilatz A, Veeratterapillay R, Köves B, et al. Update on Strategies to Reduce Infectious Complications After Prostate Biopsy. Eur Urol Focus 2019;5:20-8.

13. Puech $\mathrm{P}$, Rouvière $\mathrm{O}$, Renard-Penna $\mathrm{R}$, et al. Prostate cancer diagnosis: multiparametric MR-targeted biopsy with cognitive and transrectal US-MR fusion guidance versus systematic biopsy--prospective multicenter study. Radiology 2013;268:461-9.

14. Vickers AJ, Roobol MJ, Lilja H. Screening for prostate cancer: early detection or overdetection? Annu Rev Med 2012;63:161-70.

15. Zhao HW, Li J, Cao JZ, et al. Contrast-enhanced transrectal ultrasound can reduce collection of unnecessary biopsies when diagnosing prostate cancer and is predictive of biochemical recurrence following a radical prostatectomy in patients with localized prostate cancer. BMC Urol 2020;20:100.

16. Tan N, Lin WC, Khoshnoodi P, et al. In-Bore 3-T MRguided Transrectal Targeted Prostate Biopsy: Prostate Imaging Reporting and Data System Version 2-based Diagnostic Performance for Detection of Prostate Cancer. Radiology 2017;283:130-9.

17. Tan N, Margolis DJ, McClure TD, et al. Radical prostatectomy: value of prostate MRI in surgical planning. Abdom Imaging 2012;37:664-74.

18. Barentsz JO, Richenberg J, Clements R, et al. ESUR prostate MR guidelines 2012. Eur Radiol 2012;22:746-57.

19. Weinreb JC, Barentsz JO, Choyke PL, et al. PI-RADS Prostate Imaging - Reporting and Data System: 2015, Version 2. Eur Urol 2016;69:16-40.

20. Kasivisvanathan V, Rannikko AS, Borghi M, et al. MRITargeted or Standard Biopsy for Prostate-Cancer Diagnosis. N Engl J Med 2018;378:1767-77.

21. Schoots IG, Nieboer D, Giganti F, et al. Is magnetic resonance imaging-targeted biopsy a useful addition to systematic confirmatory biopsy in men on active surveillance for low-risk prostate cancer? A systematic review and meta-analysis. BJU Int 2018;122:946-58.

22. Buyyounouski MK, Choyke PL, McKenney JK, et al. 
Prostate cancer - major changes in the American Joint Committee on Cancer eighth edition cancer staging manual. CA Cancer J Clin 2017;67:245-53.

23. Clavien PA, Barkun J, de Oliveira ML, et al. The ClavienDindo classification of surgical complications: five-year experience. Ann Surg 2009;250:187-96.

24. Epstein JI, Allsbrook WC Jr, Amin MB, et al. The 2005 International Society of Urological Pathology (ISUP) Consensus Conference on Gleason Grading of Prostatic Carcinoma. Am J Surg Pathol 2005;29:1228-42.

25. Harnden P, Naylor B, Shelley MD, et al. The clinical management of patients with a small volume of prostatic cancer on biopsy: what are the risks of progression? A systematic review and meta-analysis. Cancer 2008;112:971-81.

26. Hansen NL, Barrett T, Kesch C, et al. Multicentre evaluation of magnetic resonance imaging supported transperineal prostate biopsy in biopsy-naïve men with suspicion of prostate cancer. BJU Int 2018;122:40-9.

27. Mannaerts CK, Kajtazovic A, Lodeizen OAP, et al. The added value of systematic biopsy in men with suspicion of prostate cancer undergoing multiparametric MRI-targeted biopsy. Urol Oncol 2019;37:298.e1-9.

28. Tonttila PP, Lantto J, Päkkö E, et al. Prebiopsy Multiparametric Magnetic Resonance Imaging for Prostate Cancer Diagnosis in Biopsy-naive Men with Suspected Prostate Cancer Based on Elevated Prostate-specific Antigen Values: Results from a Randomized Prospective Blinded Controlled Trial. Eur Urol 2016;69:419-25.

29. Ploussard G, Beauval JB, Lesourd M, et al. Added value of concomitant systematic biopsies for grade group prediction based on radical prostatectomy final pathology in magnetic resonance imaging positive cases undergoing

Cite this article as: Uno H, Taniguchi T, Seike K, Kato D, Takai M, Iinuma K, Horie K, Nakane K, Koie T. The accuracy of prostate cancer diagnosis in biopsy-naive patients using combined magnetic resonance imaging and transrectal ultrasound fusion-targeted prostate biopsy. Transl Androl Urol 2021;10(7):2982-2989. doi: 10.21037/tau-21-250 fusion targeted biopsies. J Urol 2019;202:1182-7.

30. Bjurlin MA, Meng X, Le Nobin J, et al. Optimization of prostate biopsy: the role of magnetic resonance imaging targeted biopsy in detection, localization and risk assessment. J Urol 2014;192:648-58.

31. Ahmed HU, El-Shater Bosaily A, Brown LC, et al. Diagnostic accuracy of multi-parametric MRI and TRUS biopsy in prostate cancer (PROMIS): a paired validating confirmatory study. Lancet 2017;389:815-22.

32. Johnson DC, Raman SS, Mirak SA, et al. Detection of Individual Prostate Cancer Foci via Multiparametric Magnetic Resonance Imaging. Eur Urol 2019;75:712-20.

33. Fütterer JJ, Briganti A, De Visschere P, et al. Can Clinically Significant Prostate Cancer Be Detected with Multiparametric Magnetic Resonance Imaging? A Systematic Review of the Literature. Eur Urol 2015;68:1045-53.

34. Mazzone E, Stabile A, Pellegrino F, et al. Positive predictive value of Prostate Imaging Reporting and Data System version 2 for the detection of clinically significant prostate cancer: A systematic review and meta-analysis. Eur Urol Oncol 2020;S2588-9311(20)30212-1.

35. Han C, Liu S, Qin XB, et al. MRI combined with PSA density in detecting clinically significant prostate cancer in patients with PSA serum levels of 4 10ng/mL: Biparametric versus multiparametric MRI. Diagn Interv Imaging 2020;101:235-44.

36. Zhu K, Qin Z, Xue J, et al. Comparison of prostate cancer detection rates between magnetic resonance imagingtargeted biopsy and transrectal ultrasound-guided biopsy according to Prostate Imaging Reporting and Data System in patients with PSA $\geq 4 \mathrm{ng} / \mathrm{mL}$ : a systematic review and meta-analysis. Transl Androl Urol 2019;8:741-53. 